\title{
The IPPOG Resource Database: Making particle physics outreach \& education available worldwide
}

\author{
Barbora Bruant Gulejova $^{a}$ on behalf of IPPOG Collaboration ${ }^{b}$ \\ a University of Bern, \\ Bern, Switzerland \\ b International Particle Physics Outreach Group (IPPOG), \\ CERN, Meyrin, Switzerland \\ E-mail: barbora.gulejova@cern.ch
}

The International Particle Physics Outreach Group (IPPOG) has been making concerted and systematic efforts to present and popularize particle physics across all audiences and age groups since almost 25 years. Today the scientific community has in IPPOG a strategic pillar in fostering long-term, sustainable support for fundamental research around the world. One of the main tools IPPOG has been offering to the scientific community, teachers and educators since almost 10 years is the Resource Database (RDB), an online platform containing the collection of high-quality engaging education and outreach materials in particle physics and related sciences. After almost 10 years, a new digital portfolio aiming to greatly broaden the audience type and strengthen the user experience, is being developed including a new RDB, which is currently undergoing a major curation process in order to ensure the resources are up-to-date and of the highest quality. IPPOG wants the new website to become more open to students, teachers, and the general public and the new RDB to become the primary source of particle physics outreach material in the world, which would help to bring particle physics closer to the society.

The Ninth Annual Conference on Large Hadron Collider Physics - LHCP2021

7-12 June 2021

Online 


\section{Introduction: Challenges of the particle physics community}

Threatened financial support for large experimental endeavors, falling interest of young people to engage in studies of STEM, especially physics, and mistrust in science are the main challenges the scientific community is faced with currently. These are based on the misperception of science, especially physics and basic research in society. This attitude towards physics by non-scientific audiences is largely caused by the scarce exposure of society to modern physics, which is in most cases not included in school curricula. Introducing particle physics and related sciences to students and the public, while showing the current state of art of contemporary physics and bringing the understanding of the world we live in and its technologies, is of vital importance.

Before the update of physics school curricula is achieved, the science outreach and extra-curriculum activities are the only possibility to bridge the gap between contemporary scientific research and science education at schools and thus the awareness of modern science by society.

\subsection{International Particle Physics Outreach Group}

The International Particle Physics Outreach Group (IPPOG) has been making concerted and systematic efforts to present and popularise particle physics across all audiences and age groups for more than two decades by developing suitable methods, tools and activities. Today the scientific community has in IPPOG a strategic pillar to aid the fostering of long-term, sustainable support for fundamental research around the world.

The IPPOG's principal aim is to maximise the impact of education and outreach efforts related to particle physics. It contributes to global efforts in strengthening the cultural awareness in the understanding and support of particle physics and related sciences, in raising scientific literacy in society, educating the public on the values of basic research and in developing and training the next generation of researchers, scientists and engineers. In particular, IPPOG's purpose is to raise standards of global outreach and informal science education efforts of particle physics, to communicate its results and findings to the public, to bring new discoveries in all areas of particle physics research to young people and to convey to the public that the beauty of nature is indeed becoming understandable from the interactions of its most fundamental constituents - the elementary particles.

Current IPPOG activities include the ever-growing and well-established International Masterclasses on Particle Physics programme [1], the outreach Resource Database (see chapter 2) [2], the Global Cosmic Rays experiments at schools platform currently in development [3], support for exhibitions and activities at public events and festivals (e.g. Colours of Ostrava in Czech Republic [4], Universal Science [5]), and different topical programmes and competitions targeting young and diverse audiences (like Particles4U [6], Girls, do Physics! [7], Creating Ambassadors for Science in Society [8], Cascade competitions in UK [9] and Slovakia [10] etc...). These diverse activities allow IPPOG to bridge the gap between science education at school and modern scientific research by inspiring, motivating and educating an especially young audience, offering hands-on experience and connecting physics to real life while using cutting edge technologies.

\section{IPPOG Resource Database}

The richness of IPPOG's expertise given the diversity of its members in terms of cultural and educational backgrounds, provides a perfect platform for sharing, developing and improving the explanatory and teaching materials, strategies, methods, activities and tools to reach broader audiences, based on the best practices in outreach and education of particle physics and related sciences. In its commitment to foster science dissemination, an online platform has been built to facilitate the exchange of particle physics education and outreach resources across the globe. IPPOG's Resource Database (RDB) is a collection of high quality engaging materials (e.g. videos, posters, talks, hands-on activities, tools, brochures and more) recommended by IPPOG representatives and contributors to help sharing the wonders and excitement of particle physics with teachers, students and the general public. The first version of the IPPOG's RDB was released in 2011 as the "best practice" exchange platform [11]. Almost 400 items have been collected since [12]. They are filtered by physics topics (from particles and their interactions through dark matter, extradimensions to detectors and accelerators), item types (e.g. video, poster, lesson plan, etc.), target audiences and language. 


\subsection{New IPPOG Website}

After almost 10 years, IPPOG has embarked on an ambitious project to improve the user experience across the IPPOG digital portfolio (websupportite and social media channels) and to strengthen the IPPOG brand online by creating a new website (see Fig.1) including a new RDB (see Fig. 2). The goal of the new design is to greatly broaden the audience type and use of the web pages and available resources. IPPOG wants the new website to become more open to students, teachers and the general public, and for the RDB to become the primary source of particle physics outreach material in the world [2].

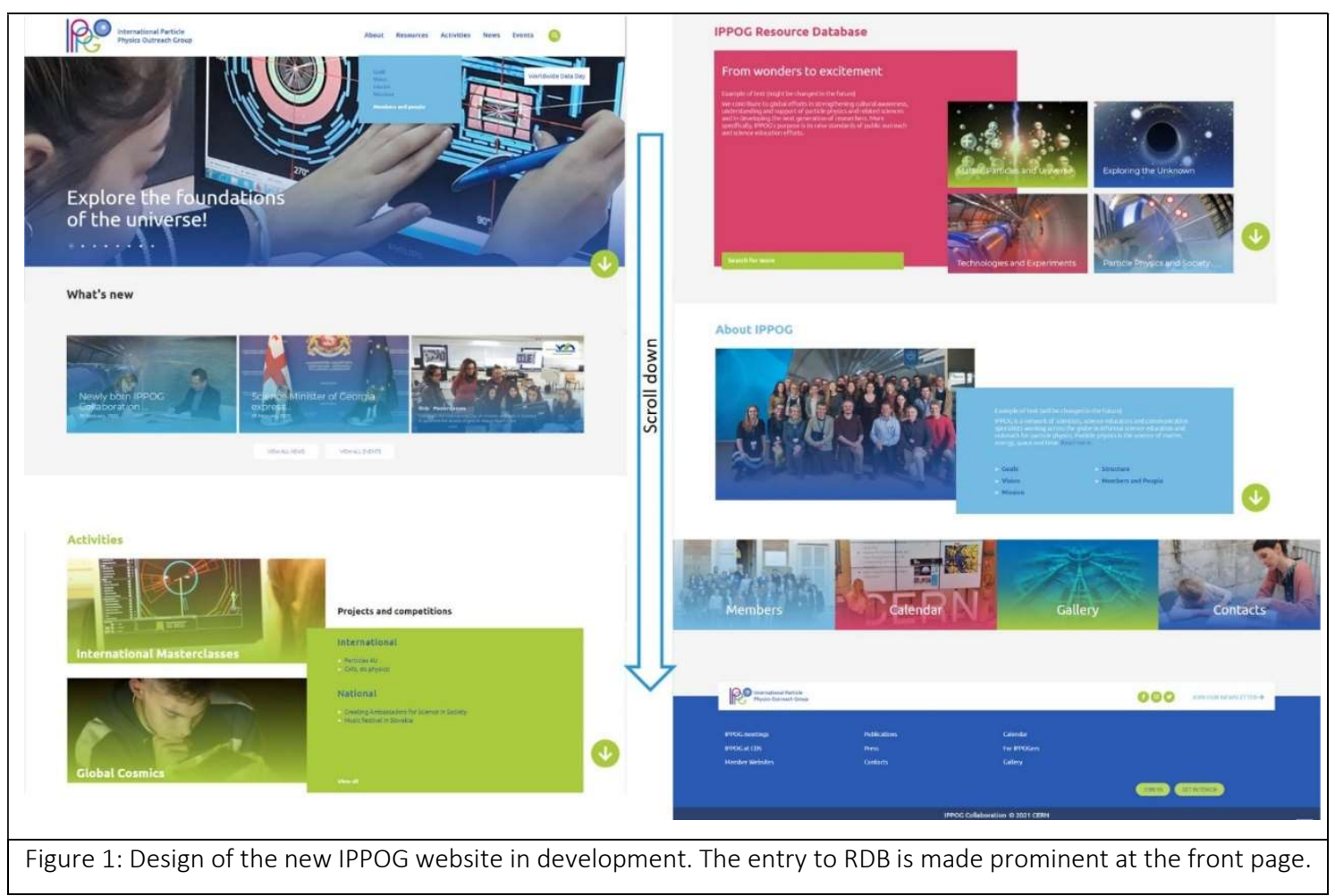

\subsection{New IPPOG Resource Database}

For several years discussions within IPPOG were considering how to improve the functionality, keep the content up-to-date and provide the full range coverage of relevant physics topics. As a result new RDB has been developed in close collaboration with IPPOG community and its target audiences (especially high school physics teachers) offering more user-friendly way of navigating, search, submissions and feedback $[13,14]$. As shown on Fig.2, one can search by a filter, but also use the quick search by topics by clicking directly on the images on the top, while at every step there is a possibility to refine the search using the filter on the side, which always remains in view.

IPPOG aims the information in RDB to be readily understandable and regularly updated to reflect the latest discoveries in particle physics. In 2020 an official RDB curation group, including teachers, scientists and science educators from over 15 countries, 3 experiments and CERN, has been created with mandate to review nearly 400 resources collected over past 10 years to ensure that these are still correct and useful and to assign new tags following new taxonomy. A new tag 'school topic' has been added, which helps teachers to identify where in their classical physics curriculum they can use the particular resource. Many new items are being collected at the same time in order to provide the most comprehensive and up-to-date collection of outreach and educational materials in particle physics and related sciences worldwide. Even though most of the resources are in English, thanks to the large international representation of IPPOG, the materials span 24 languages. New content for the RDB is being prepared for the future, with the aim to provide a powerful tool for the scientific community to shape the attitude and perception of physics and fundamental research by decision makers, funding bodies and the public, and to motivate young people to undertake physics studies. 


\subsection{Target audiences: who can find what?}

The first primary group of IPPOG RDB users (particle physics and scientific community active in outreach and education) are expected to seek the materials in support of their own particle physics outreach projects and inspiration (e.g. for public talks). The second primary group, teachers and educators, are expected to find the extra-curricular activities, lesson plans or projects for the classroom, and even the materials to inspire young students and motivate them to study STEM subjects and become scientifically aware citizens. Curious students will find materials or activities in particle physics and related sciences complementary to what they are being taught at school. More details on the subject can be found in [14].

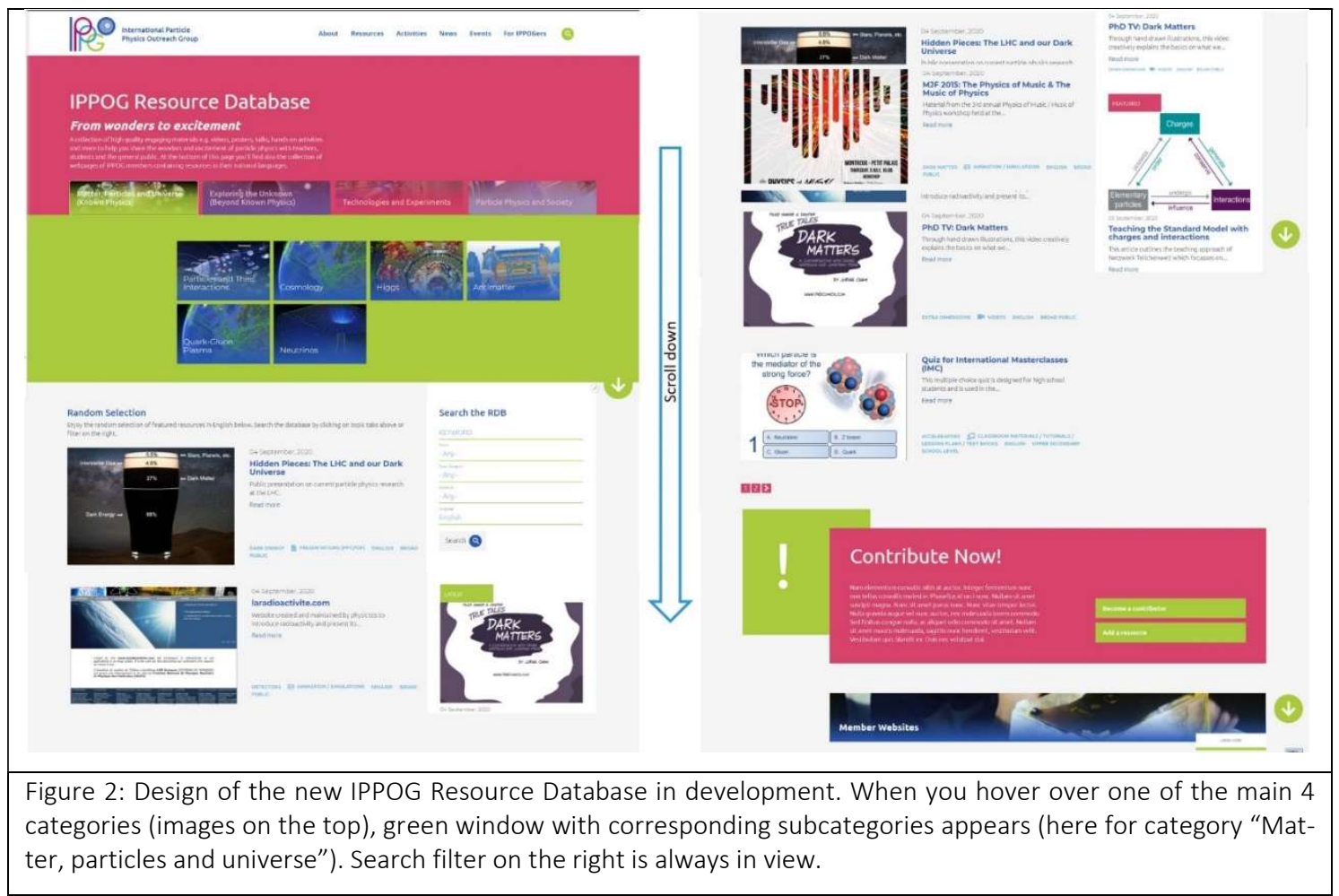

\section{References}

[1] IPPOG Physics Masterclasses, http://physicsmasterclasses.org/

[2] Barbora Bruant Gulejova, IPPOG: Bridging the gap between science education at school and modern scientific research, 2019, https://cds.cern.ch/record/2746338? ln=en

[3] Barbora Bruant Gulejova, IPPOG Global Cosmic Rays Portal: Making Cosmic Rays Studies available to schools worldwide, Proceedings ICRC 2021, https://cds.cern.ch/record/2775675? $\ln =\mathrm{en}$

[4] Colours of Ostrava, Music Festival in Czech Republic, https://www.colours.cz/

[5] Universal Science Festival, https://universalscience.web.cern.ch/

[6] Particles4U, IPPOG competition 2018, http://ippog.org/particles4u

[7] Girls, do physics!, IPPOG campaign and competition, https://ippogorg.wixsite.com/girlsdophysics

[8] Creating Ambassadors for Science in Society event: http://indico.cern.ch/event/736469/

[9] Cascade Competitions UK, https://www.birmingham.ac.uk/schools/physics/outreach/secondaryschools/cascade.aspx

[10] Cascade Competitions Slovakia, https://fyzika.uniza.sk/cascade/

[11] L. McCarthy, M. Kobel, D. Barney, EPPOG à IPPOG: An Evolution of Particle Physics Outreach, 2010, https://indico.cern.ch/event/107976/contributions/1309725/attachments/24972/35951/E PPOG_IPPOG_MKandLMandDBv4.pdf

[12] IPPOG Resource Database: http://ippog.org/resources

[13] B. Bruant Gulejova, New IPPOG Website Design and Development, Technical Specification, 2019, https://cds.cern.ch/record/2719227

[14] Barbora Bruant Gulejova, IPPOG: Bridging the gap between science education at school and modern scientific research, 2019, https://cds.cern.ch/record/2746338? $1 \mathrm{n}=\mathrm{en}$ 